\title{
Impact of hypertension on somatic pain sensitivity in chronic headache
}

\author{
ET Tafuri ${ }^{*}$, S Di Fabio ${ }^{2}$, E Cozza ${ }^{2}$, GP Affaitati ${ }^{1}$, A Fabrizio ${ }^{2}$, C Tana ${ }^{2}$, L Felicioni ${ }^{2}$, M Bucci ${ }^{3}$, A Mezzetti ${ }^{3}$, \\ MA Giamberardino ${ }^{2}$
}

From The European Headache and Migraine Trust International Congress

London, UK. 20-23 September 2012

\section{Aim of investigation}

Previous studies have shown that chronic headache (migraine and/or tension-type; $\mathrm{CH}$ ) is characterized by diffuse somatic hyperalgesia, while arterial hypertension (HY) produces somatic hypoalgesia which is only partly attenuated by antihypertensive treatment. The aim of the study was to assess if comorbidity between $\mathrm{CH}$ and $\mathrm{HY}$ results into an attenuation of the hyperalgesia due to headache.

\section{Methods}

Forty-eight patients of both sexes [28-56 years] with moderate essential hypertension plus chronic headache $(\mathrm{HY}+\mathrm{CH})$ were examined. Twenty-two received no treatment for hypertension, the remaining 26 were under therapy, with good control of pressure values. All underwent measurement of a) blood pressure at rest; b) pressure and electrical pain thresholds in the trapezius, deltoid and quadriceps muscle of one side. The results in this group were compared with those of 40 healthy control subjects (C), 52 patients with chronic headache without hypertension $(\mathrm{CH})$ and 190 patients with hypertension without headache (82 without and 108 with treatment)(HY)(all age and sex-matched).

\section{Results}

Immediately before threshold evaluation, untreated hypertensive patients had higher than normal blood pressure levels, while hypertensive patients under treatment, headache patients without hypertension and control subjects had normal blood pressure values. Pain thresholds at all sites in $\mathrm{HY}+\mathrm{CH}$ were significantly lower than normal and HY in both treated and untreated patients $(\mathrm{p}<0.001)$. They were not significantly different from thresholds recorded in $\mathrm{CH}$.

\section{Conclusion}

Comorbidity between chronic headache and arterial hypertension (with or without antihypertensive treatment) does not involve any attenutation of the typical diffuse hyperalgesia that characterizes chronic headache. These results suggest that the sensitization process behind diffuse hyperalgesia in chronic pain forms such as chronic headache prevails on the hypoalgesia-determining mechanisms of hypertension in comorbid patients.

\section{Author details}

${ }^{1}$ Headache Center and Center for the Study of Hypertension, G. D'Annunzio University of Chieti, Italy. ${ }^{2}$ Headache Center, G. D'Annunzio University of Chieti, Italy. ${ }^{3}$ Center for the Study of Hypertension, G. D'Annunzio University of Chieti, Italy.

\section{Published: 21 February 2013}

\section{References}

1. France $C R$, al'absi $M$, Ring $C$, France JL, Brose J, Spaeth D, Harju A Nordehn G, Wittmers LE: Assessment of opiate modulation of pain and nociceptive responding in young adults with a parental history of hypertension. Biol Psychol 2005, 70:168-174.

2. France CR, Froese SA, Stewart JC: Altered central nervous system processing of noxious stimuli contributes to decreased nociceptive responding in individuals at risk for hypertesnion. Pain 2002, 98:101-108.

doi:10.1186/1129-2377-14-S1-P160

Cite this article as: Tafuri et al:: Impact of hypertension on somatic pain sensitivity in chronic headache. The Journal of Headache and Pain 2013 14(Suppl 1):P160. 